\title{
Ampliação da distribuição geográfica do caranguejo de água doce Trichodactylus petropolitanus (Goeldi, 1886) (Decapoda, Trichodactylidae) através do registro de ocorrência na cidade de Mendes, Rio de Janeiro, Brasil
}

Expansion of the geographical distribution of the freshwater crab Trichodactylus petropolitanus (Goeldi, 1886) (Decapoda, Trichodactylidae) through registration of occurrence in the city of Mendes, Rio De Janeiro, Brazil

\section{Guilherme Pinheiro Furusawa*广 e Paulo Cesar Rodrigues Cassino}

Como citar esse artigo. Furusawa, G.P.; Cassino, P.C.R. Ampliação da distribuição geográfica do caranguejo de água doce Trichodactylus petropolitanus (Goeldi, 1886) (Decapoda, Trichodactylidae) través do registro de ocorrência na cidade de Mendes, Rio de Janeiro, Brasil. Revista Teccen. 2017 Jun./Dez.; 10 (1): 31-34.

\begin{abstract}
Resumo
Os caranguejos de água doce congregam um grupo de crustáceos da família Trichodactylidae, cujas espécies desempenham importante papel na manutenção de diversos ecossistemas nos ambientes continentais, atuando em diferentes níveis tróficos, servindo ainda de recurso alimentar para muitas espécies de peixes, aves, répteis e mamíferos, incluindo o próprio homem, além da reconhecida capacidade de reciclar a matéria orgânica nos ambientes em que vivem. Toda essa importância implica na necessidade de estudos acerca destas relações, assim como da sua distribuição geográfica. Entretanto, são insipientes os estudos acerca da ecologia destes organismos no ambiente Neotropical e em destaque ao Brasil, frente principalmente a grande diversidade de ambientes nesse território. O trabalho em tela visa colaborar com a ampliação da distribuição geográfica do gênero Trichodactylus com a observação do caranguejo dulcícola Trichodactylus petropolitanus (Goeldi, 1886) (Decapoda, Trichodactylidae) na cidade serrana de Mendes, mesorregião metropolitana do Estado do Rio de Janeiro, Brasil, constituindo-se no primeiro registro desta espécie na região.
\end{abstract}

Palavras-Chave: Caranguejo de água doce; Decápodes; Mendes; Novo registro.

\begin{abstract}
Freshwater crabs congregate a group of crustaceans of Brachyura infraorder, Trichodactylidae family, whose species play an important role in the maintenance of different ecosystems in continental environments, acting in different trophic levels, Serving as food resources for many species of fish, birds, reptiles and mammals Including the human being, through the famous ability to recycle the organic matter in the environments in which they live. All this importance implies the necessities for studies about these relations like its geographical distribution. However, studies on the ecology of these organisms in the Neotropical environment are incipient. In particular Brazil, mainly due to the great diversity of environments in this territory. This work aims to collaborate with the expansion of the geographic distribution of the genus Trichodactylus. With the observation of the freshwater crab Trichodactylus petropolitanus (Goeldi, 1886) (Decapoda, Trichodactylidae) in the city of Mendes, metropolitan mesoregion of the State of Rio de Janeiro, Brazil, constituting the first record of this species in the region.
\end{abstract}

Keywords: Brachyura, Decapoda, Mendes city, New record, Trichodactylidae.

\section{Introdução}

Os caranguejos dulcícolas são crustáceos da infraordem Brachyura, de grande importância ecológica e econômica (Magalhães, 2003), além de servirem como bioindicadores da qualidade ambiental (Helawell, 1978). Estima-se que em todo o mundo existam, até o momento, aproximadamente 1.476 espécies descritas, distribuídas em 238 gêneros, organizados dentro de oito famílias, estabelecidas em quatro superfamílias: Gecarcinucoidea, Potamoidea, Pseudothelphusoidea e Trichodactyloidea (Yeo et al., 2008). No Brasil, até o momento, são conhecidas 45 espécies, pertencentes às superfamílias Pseudothelphusoidea e Trichodactyloidea (Magalhães, 2003).

Os representantes da família Trichodactylidae ocorrem em rios e riachos montanhosos das Américas do Sul e Central (Magalhães, 2003). No Brasil, o gênero Trichodactylus (Latreille, 1828) é representado por nove

Afiliação dos autores:

$\dagger$ Universidade Federal Rural do Rio de Janeiro, CPGCV, IZMA - Instituto Zoobotânico de Morro Azul, RJ.

* Universidade Severino Sombra, Vassouras, RJ.

E-mail para correspondência: *gfurusawa@gmail.com 
espécies (Mossolin \& Mantelatto, 2008). Trichodactylus petropolitanus (Goeldi, 1886) pode ser encontrado nos estados de Minas Gerais, Rio de Janeiro, São Paulo, Paraná e Santa Catarina, sendo amplamente distribuído nos rios e riachos montanhosos de remanescentes de floresta Atlântica (Vilas Boas et al., 2013), e até recentemente esta espécie era considerada endêmica do Brasil, entretanto foi registrada no nordeste da Argentina (César et al., 2003). Esta espécie apresenta habito onívoro, sendo pouco exigente quanto sua alimentação (Venâncio, 2005), o que lhe confere importante papel na cadeia trófica.

Acerca de sua variação altitudinal, $T$. petropolitanus é mais comumente registrado em altitudes inferiores a 300 metros (Magalhães, 1999), ainda que haja registros recentes da distribuição desta espécie em diversas altitudes, como a aproximadamente 390 metros no rio Paraíba do Sul, Cantagalo, Rio de Janeiro (APARPS, 2013), cerca de 500 metros de altitude na região da Bacia do Ribeira de Iguape (Rocha \& Bueno, 2004) e 640 metros no Córrego da Mina, zona rural de Caçapava (Venâncio op cit.), estes últimos no Estado de São Paulo. Em 850 metros na cidade de Piranguinho, região da bacia do alto Rio Sapucaí, Minas Gerais (Vilas Boas op cit.) e acima de 900 metros no Rio Verde na região do sul de Minas Gerais, próximo ao município de Soledade de Minas (Oliveira \& Camargo, 2012).

Apesar de sua importância ecológica, ainda pouco se conhece sobre a biologia de caranguejos dulcícolas no Brasil (Rocha \& Bueno, 2004). Nesse sentido, Magalhães (1999) e Venâncio \& Leme (2010) alertam para a importância da obtenção de conhecimentos mais detalhados acerca da riqueza de espécies desse grupo, considerando que modificações ambientais verificadas em várias bacias, devido à pressão antrópica, podem causar alterações na comunidade biológica, inclusive na de crustáceos decápodes, que podem desaparecer completamente. Dessa forma, observamos que para se preservar uma espécie é necessário conhecer sua distribuição geográfica, pois novas ocorrências auxiliam no entendimento de seu ciclo de vida e dispersão populacional (Góes \& Fernandes-Góes, 2007).

O objetivo deste estudo foi registrar a ocorrência de $T$. petropolitanus na cidade serrana de Mendes - Rio de Janeiro, contribuindo para a compreensão da distribuição desta espécie, assim como colaborar na elaboração de um plano de manejo que vise a conservação destes organismos.

\section{Material e métodos}

A cidade de Mendes é situada após a escarpa da Serra do Mar na borda do planalto fluminense, inserida na bacia hidrográfica do Rio Paraíba do Sul, região Centro Sul fluminense, com clima Tropical de Altitude Cwa, entre as coordenadas: latitude $22^{\circ} 31^{\prime} 36^{\prime \prime}$ sul e longitude $43^{\circ} 43^{\prime} 58^{\prime}$ oeste (Figura 1), com altitude de até 446 metros.

O local onde os espécimes foram observados encontra-se na área central desta cidade em um pequeno lago artificial com aproximadamente 36 metros quadrados, com até $30 \mathrm{~cm}$ de profundidade, inserido em uma propriedade particular, dista cerca de 20 metros do Rio Sacra Família.

A coleta foi realizada no mês de novembro de 2014, de forma manual e noturna, único horário onde estes espécimes foram observados em atividade, sendo sacrificado e conservado em

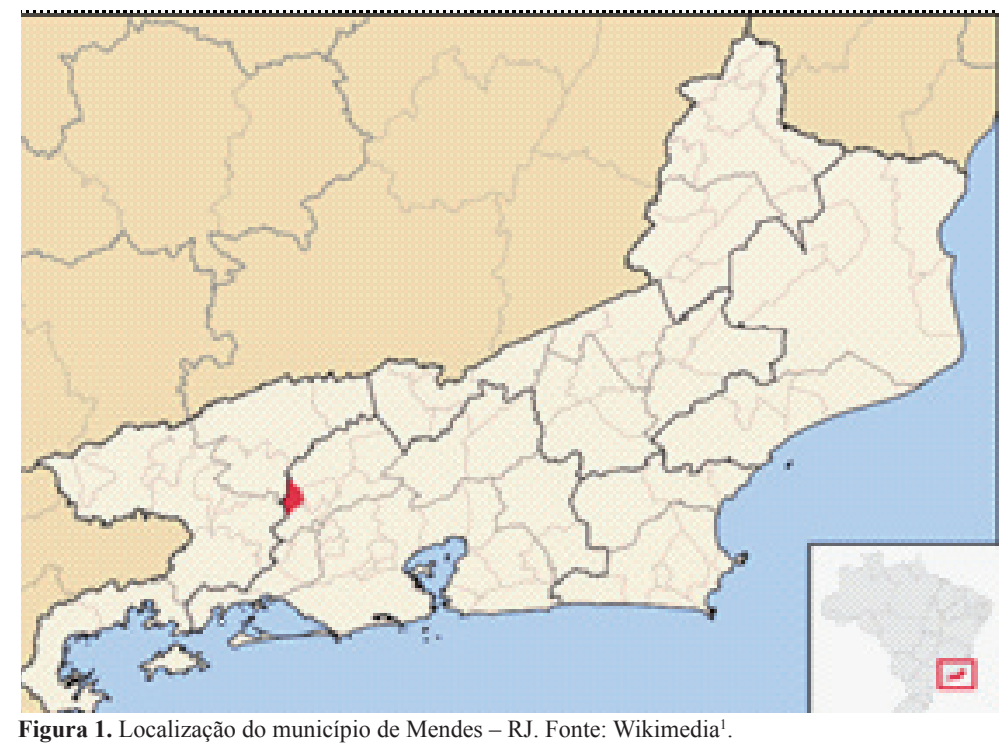

${ }^{1}$ Disponível em $<$ https://commons.wikimedia.org/wiki/File:RiodeJaneiro_Municip_Mendes.svg $>$ 
Álcool etílico a 96\%. Posteriormente foi enviado ao acervo do Museu de Zoologia da USP, sob os cuidados do professor Marcos Tavares, curador.

\section{Resultados e discussões}

O espécime coletado foi uma fêmea identificada como Trichodactylus petropalitanus (Goeldi, 1886) (Crustacea: Decapoda: Brachyura: Trichodactylidae) (Figura 2).

$\mathrm{O}$ registro de $T$. petropolitanus na cidade de Mendes constitui-se no primeiro registro desta espécie nesta região fluminense, colaborando com estudos biogeográficos que se constituem numa importante ferramenta para o conhecimento e o entendimento dos processos de distribuição da fauna de caranguejos dulcícola no Brasil. Este registro visa ainda contribuir para o desenvolvimento de ações para conservação desta espécie na região.

Vale ressaltar que, sendo este pequeno lago próximo cerca de 20 metros do Rio Sacra Família, o mais importante rio que cruza esta cidade, não ignoramos a possibilidade da existência desta espécie neste rio.

Não obstante, o proprietário da área onde se encontra esta pequena população, deseja, em futuro próximo, aterrar o lago artificial visando ampliar sua área construída. Assim, estuda-se o transporte desta população para uma unidade de conservação próxima objetivando a manutenção desta espécie na região.

\section{Conclusão}

Concluímos que, com a presente nota, registrase a ampliação da distribuição geográfica do gênero
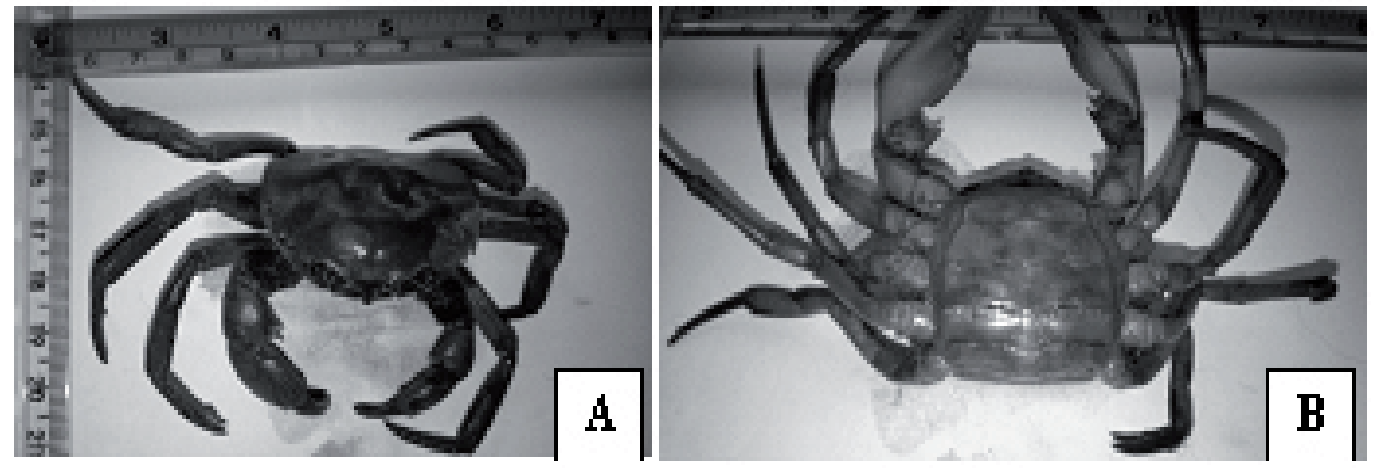

Figura 2. Trichodactylus petropolitanus (Goeldi, 1886). Fêmea adulta, em vista dorsal (A) e em vista ventral (B). Espécime obtido durante coleta em Mendes, Rio de Janeiro. Fonte: Arquivo pessoal.

Trichocadtylus, registrando que Trichodactylus petropalitanus (Goeldi, 1886) ocorre na cidade de Mendes, Rio de Janeiro, e observamos a necessidade de um plano de manejo para a manutenção desta espécie na região.

\section{Referências}

APARPS - Associação de Pescadores e Amigos do rio Paraíba do Sul. (2013). Projeto Piabanha. $3^{\circ}$ Relatório: Monitoramento biológico de espécies aquáticas ameaçadas de extinção na bacia do rio Paraíba do Sul: desenvolvimento de sistema piloto e implementação de plano de ação. PESAGRO/RJ, Rio de de Janeiro.

César, I. I., Armendáriz, L. C., Becerra, R. V., \& Liberto, R. (2004). Biodiversidad de Crustácea (Anostraca, Notostraca, Spinicaudata,

Laevicaudata, Ostracoda, Amphipoda y Brachyura Trichodactylidae) de la Mesopotamia argentina. Insugeo, Miscelánea, 12: 247 - 252.

Góes, J. M., \& Fernandes-Góes, L. C. (2007). First occurrence of the crab Calappanitida Holthuis, 1958 (Brachyura, Calappidae, Calappinae) on the coast of the state of Piauí, Brazil. Biotemas, Florianopolis, 20 (1): 123-125.
Gomides, S. C., Novelli, I. A.; Santos, A. O., Brugiolo, S. S. S., \& Sousa, B. M. (2009). Novo registro altitudinal de Trichodactylus fluviatilis (Latreille, 1828) (Decapoda, Trichodactylidae) no Brasil. Acta Scientiarum. Biological Sciences, Maringá, v. 31, n. 3, p. 327-330.

Helawell J. M. (1978). Biological surveillance of rivers. Stevenage, England: Water Research Centre. Limnology and Oceanography, 24(4), 793-794.

Magalhães, C. (1999). Família Trichodactylidae (caranguejos braquiúros da água doce) In: Buckup, L.; Bond-Buckup, G. (Ed.). Os crustáceos do Rio Grande do Sul. Porto Alegre: UFRGS, p. 486-490.

Magalhães, C. (2003). Famílias Pseudothelphusidae e Trichodactylidae. Pp. 143-287. In: MELO, G.A.S. (Ed). Manual de identificação dos crustacea decapoda de água doce do Brasil. Editora Loyola, São Paulo.

Mossolin, E. C., \& Mantelatto, F. L. (2008). Taxonomic and distributional results of a freshwater crab fauna survey (Family Trichodactylidae) on São Sebastião Island (Ilhabela), South Atlantic, Brazil. Acta Limnologica Brasiliensia, 20(2): p. 125-129.

Oliveira, R. A., \& Camargo, F. V. (2012). Aumento na distribuição altidudinal de Trichodactylus petropolitanus (Goeldi, 1886) (Decapoda, Trichodactylidae) no Brasil. Anais do III Congresso IC FEPI. 
Rocha, S. S., \& Bueno, S. L. S. (2004). Crustáceos decápodes de água doce com ocorrência no Vale do Ribeira de Iguape e rios costeiros adjacentes, São Paulo, Brasil. Revista Brasileira de Zoologia, v. 21, n. 4, p. 1001-1010.

Venâncio, F. A. (2005). Biologia populacional do caranguejo de água doce Trichodactylus petropolitanus (Goeldi, 1886) (Crustácea, Brachyura, Trichodactylidae) no córrego da Mina, Caçapava, São Paulo: monitoramentoambiental a partir de estudo de populações animais. 2005.

62f.:il.. (Dissertação de Mestrado em Ciências Ambientais) Universidade de Taubaté, Programa de Pós graduação em Ciências Ambientais, Taubaté, São Paulo.

Venâncio, F., \& Leme, M. A. (2010). The freshwater crab Trichodactylus petropolitanus (Goeldi, 1886) (Decapoda, Trichodactylidae) associated with roots of Hedychium coronarium Koenig (Zingiberacea) Pan-American Journal of Aquatic Sciences, 5(4):501-501.

Vilas Boas, A. H., Camargo, F. V., Albo, R., \& Sene, J. L. (2013). Aumento da distribuição altitudinal e geográfica de Trichodactylus

petropolytanus (Goeldi, 1886) (Decapoda, Trichodactylidae) no Brasil. Anais do X Congresso Nacional de Meio Ambiente de Poços de Caldas, V.5, N.1, Poços de Caldas, MG.

Yeo, D. J. C., Ng, P. K. L., Cumberlidge, N., Magalhães, C., Daniels, S. R., \& Campos, M. R. (2008). Global diversity of crabs (Crustacea: Decapoda: Brachyura) in freshwater. Hydrobiologia, 595: p. 275-286. 